\title{
Effect of cholinergic and anticholinergic agents on tardive dyskinesia ${ }^{1}$
}

\author{
H. L. KLAWANS ${ }^{2}$ AND R. RUBOVITS \\ From the Division of Neurology, Michael Reese Medical Center, Chicago, Illinois \\ and the Department of Psychiatry, University of Maryland, Baltimore, Maryland, U.S.A.
}

SYNOPSIS Tardive dyskinesia, like several other choreiform disorders, is felt to be primarily related to dopaminergic activity within the striatum. Physostigmine has been demonstrated to improve the abnormal movements in patients with tardive dyskinesia while scopolamine tends to aggravate abnormal movements and in some cases elicits abnormal movement not previously observed. This evidence supports the hypothesis that anticholinergic therapy in patients prone to develop tardive dyskinesia may increase the incidence of this disorder by lowering the threshold for the appearance of these movements.

Tardive dyskinesia is a well-recognized sideeffect of long-term neuroleptic therapy (Crane, 1968). The most prominent manifestation is lingual-facial-buccal dyskinesia. Limb and trunkal chorea may accompany the facial movements (Paulson, 1968). The syndrome is most often seen in patients ranging in age from 50 to 70 years who are most often diagnosed as suffering chronic deteriorating schizophrenia. Tardive dyskinesia occurs late in the course of neuroleptic therapy, often after a decrease in the drug dosage or discontinuation of the therapy. The involuntary facial movements often persist for months to years after the neuroleptic treatment is discontinued, and the response to any type of therapy is poor (Crane, 1968; Delay and Deniker, 1969; Faurbye, 1970).

The lingual-facial-buccal masticatory syndrome is not unique to tardive dyskinesia. The syndrome was first noted as an integral part of the hyperkinesia of Huntington's chorea (Huntington, 1872). It occurs also as a side-effect of the long-term, high dose L-dopa therapy of Parkinson's disease (Cotzias et al., 1967).

The pathophysiology and pathogenesis of lingual-facial-buccal dyskinesias have not yet

\footnotetext{
1 This work was supported in part by a grant from the United Parkinson Foundation, Chicago, Illinois, U.S.A.

2 Address for reprints: Harold L. Klawans, Division of Neurology, Michael Reese Medical Center, Chicago, Illinois 60616, U.S.A.
}

been fully elucidated. However, there is evidence which suggests that dopamine acting at striatal dopaminergic receptor sites may be closely related to the initiation of these choreiform movements in several clinical settings. Drugs which alter the availability of dopamine at dopaminergic receptor sites alter choreiform symptomatology. Huntington's chorea is relieved by drugs which decrease the activity of dopamine at striatal dopamine receptors, while L-dopa, which markedly increases available dopamine, exacerbates the symptomatology of Huntington's chorea (Klawans, 1970, 1973a).

L-dopa-induced dyskinesias are also related to the activity of dopamine at dopaminergic receptors in the striatum. It has been suggested that the prolonged dopaminergic denervation of the striatum characteristic of Parkinson's disease may induce cellular changes that produce a type of dopaminergic denervation hypersensitivity which is related to the appearance of L-dopa-induced lingual-facial-buccal dyskinesia (Klawans et al., 1970).

Tardive dyskinesia is also thought to be related to dopaminergic mechanisms. The neuroleptic agents which are felt to be responsible for the production of tardive dyskinesia are known to block the access of dopamine to striatal dopaminergic receptor sites (van Rossum, 1967) and, as a result, produce the symptoms of Parkinson- 
ism (Klawans, 1968). It has been proposed that the neuroleptic blockade of dopamine receptors may be the equivalent of a 'chemical denervation' of those dopamine-sensitive cells (Klawans, 1973b). This chemical denervation may, in a fashion analogous to the pathological anatomical denervation of idiopathic Parkinson's disease, induce alterations in the dopamine-sensitive cells such that, when dopamine access is restored by the diminution or removal of the chemical barrier, an abnormal cellular responsiveness to the dopamine results in the abnormal clinical features of lingual-facial-buccal dyskinesia (Rubovits and Klawans, 1972; Klawans, 1973b).

Although the pathophysiology of lingualfacial-buccal dyskinesias seems to be most closely related to dopaminergic mechanisms in the striatum, other neuronal systems, particularly cholinergic pathways, also influence striatal function. Acetylcholine has been shown to exert an influence on the striatum opposite to that of dopamine, and a balance of influence of the two neurotransmitters on the striatum is thought to be necessary for normal function (Klawans, 1968, 1973a). Since acetylcholine and dopamine have opposite effects on the striatum and a balance is necessary for normal striatal function, it is reasonable that manipulation of the effect of one system would modify the effect of the other. That manipulation of the cholinergic system can alter the symptomatology of diseases in which the pathophysiology relates most directly to dopaminergic mechanisms has been demonstrated in Parkinson's disease and Huntington's chorea.

It is most significant that cholinergic and anticholinergic agents have been shown to modify the hyperkinesias of Huntington's chorea (Klawans and Rubovits, 1972). Physostigmine which increases the levels of acetylcholine available to act upon the striatum relieves choreiform symptoms while benztropine, an anticholinergic agent, intensifies choreiform symptomatology. It appears then that manipulation of the cholinergic system significantly influences the symptomatology of the choreatic movements in a disease (Huntington's chorea) in which the underlying pathophysiology seems to be more directly related to dopaminergic mechanisms. Tardive dyskinesia is thought to be related to an abnormal influence of dopamine on striatal dopaminer-
TABLE 1

DATA ON SUBJECTS STUDIED

\begin{tabular}{|c|c|c|c|c|c|}
\hline $\begin{array}{l}\text { Sub- } \\
\text { ject }\end{array}$ & $\begin{array}{l}\text { Age } \\
(y r)\end{array}$ & $\operatorname{Sex}$ & Diagnosis & $\begin{array}{c}\text { Dura- } \\
\text { tion } \\
(y r)\end{array}$ & Manifestation \\
\hline 1 & 4.7 & $\mathbf{F}$ & SCZ & 4 & LFB \\
\hline 2 & 61 & $\mathbf{F}$ & SCZ & 6 & LFB \\
\hline 3 & 39 & $\mathbf{M}$ & $\mathrm{SCZ}$ & 3 & $\mathbf{L F B}+\mathrm{UE}+\mathbf{L E}$ \\
\hline 4 & 46 & $\mathbf{F}$ & SCZ & 7 & $\mathbf{L F B}+\mathbf{U E}$ \\
\hline 5 & 58 & $\mathbf{M}$ & SCZ & 9 & LFB + LE + trunk \\
\hline 6 & 63 & $\mathbf{M}$ & SCZ & 4 & LFB \\
\hline 7 & 71 & $\mathbf{F}$ & $\begin{array}{l}\text { CBS with para- } \\
\text { noid features }\end{array}$ & 6 & $\mathrm{LFB}+\mathrm{UE}+\mathrm{LE}$ \\
\hline 8 & 68 & $\mathbf{M}$ & CBS & 3 & LFB + UE \\
\hline 9 & 48 & $\mathbf{F}$ & SCZ & 4 & LFB + trunk \\
\hline 10 & 56 & $\mathbf{F}$ & $\mathrm{SCZ}$ & 6 & LFB \\
\hline 11 & 52 & $\mathbf{M}$ & Chronic anxiety & 9 & $\mathrm{LFB}+\mathrm{UE}+\mathrm{LE}+$ trunk \\
\hline 12 & 61 & $\mathbf{F}$ & Chronic anxiety & 8 & $\mathrm{LFB}+\mathrm{UE}$ \\
\hline
\end{tabular}

SCZ: Schizophrenia. CBS: Chronic brain syndrome. LFB: Lingualfacial-buccal dyskinesia. UE: Upper extremity choreatic movement. $\omega$ LE: Lower extremity choreatic movement.

gic receptors. The striatal cells, as a result of prot longed chemical denervation, may be over of $\doteq$ abnormally responsive to dopamine. Thus functional imbalance between the cholinerg业 and dopaminergic systems is established. Thi study was undertaken to investigate the influence of cholinergic and anticholinergic agents patients with tardive dyskinesias. It was pre dicted that, as in Huntington's chorea, cholinefgic agents would tend to restore the functional balance of the neurotransmitters and alleviate the symptoms and that anticholinergic agents would worsen the abnormal movements.

\section{METHODS}

The subjects included in this study were 12 patients clinically diagnosed as having tardive dyskinesia (Table 1). The patients had been treated with neuroleptic agents for three to nine years, with an average of 5.75 years of therapy. Seven subjects were female, five were male, ranging in age from 39 to 71 , with an average of 55.8 years. The average ages and durations of neuroleptic therapy did not differ between the sexes in our sample. Eight patients had been $ᄋ$ diagnosed as chronic schizophrenics, two as having a chronic brain syndrome, and two as having chronic $\frac{7}{0}$ anxiety. In all subjects, lingual-facial-buccal dyskinesias were the most prominent manifestation of $\tilde{N}$ tardive dyskinesia; in three cases it was the only $N$ symptom. The remaining nine cases demonstrated limb chorea and/or trunkal movement abnormalities $\omega$ in addition to the lingual-facial-buccal dyskinesias 
TABLE 2

EFFECT OF DRUGS ON DURATION OF TONGUE EXTENSION

\begin{tabular}{|c|c|c|c|c|}
\hline \multirow[t]{2}{*}{ Drug } & \multirow{2}{*}{$\begin{array}{c}\text { Number } \\
\text { tested }\end{array}$} & \multicolumn{2}{|c|}{ Duration of tongue extension } & \\
\hline & & $\begin{array}{l}\text { Before } \\
(s)\end{array}$ & $\begin{array}{l}\text { After } \\
(s)\end{array}$ & \\
\hline Physostigmine & 12 & $\begin{array}{c}11 \cdot 4 \\
\text { (range 4-28) }\end{array}$ & $\begin{array}{c}23 \cdot 4 \\
\text { (range } 11-30 \text { ) }\end{array}$ & $P<0.05$ \\
\hline Edrophonium & 4 & $\begin{array}{c}13 \cdot 9 \\
\text { (range 6-20) }\end{array}$ & $\begin{array}{c}12 \cdot 1 \\
\text { (range 4-18) }\end{array}$ & \\
\hline Scopolamine & 4 & $\begin{array}{c}18 \cdot 4 \\
\text { (range } 16-30 \text { ) }\end{array}$ & $\begin{array}{c}6 \cdot 3 \\
\text { (range } 3-15 \text { ) }\end{array}$ & $P<0.05$ \\
\hline
\end{tabular}

(Table 1). Eight patients were receiving neuroleptic medication when tested in this study, four (cases 5, 8, 11 , and 12) were not. At the time they were studied none of the patients had drug-induced Parkinsonism.

Each patient was informed as to the nature of the two agents to be used, physostigmine and scopolamine, and the possible side-effects. They were told that this study was being carried out to increase our understanding of tardive dyskinesia and that the medications might make the abnormal movements worse or better or might have no effect. The patients were never told which medication they were being given, nor what effect the specific medication might have on their symptoms.

All medications were given intravenously. Individual injections consisted of $1.0 \mathrm{mg}$ physostigmine or $1.0 \mathrm{mg}$ scopolamine; $1.0 \mathrm{mg}$ methyscopolamine was given at the same time as physostigmine to block the peripheral muscarinic effects (Duvoisin, 1967; Klawans and Rubovits, 1972). Injections of $10 \mathrm{mg}$ edrophonium were also given to four patients. Several parameters were observed during the course of each drug trial. Each parameter was recorded at least twice before any single trial. The control level represents an average of these.

UPPER EXTREMITY CHOREA A small pocket flashlight with a coloured filter over the beam was attached to each of the patient's hands. The patient was seated in a darkened room and instructed to hold his hands out in front of him and to keep his arms and hands as still as possible. A 20-second time exposure photograph was then taken. This was repeated after a 30 -second rest period.

DURATION OF TONGUE EXTENSION The patient's ability to keep his tongue protruded was observed by instructing the patient to keep his mouth open and his tongue protruded as long as he could. The time between extension and retraction of the tongue inside the inner border of the lower lips was recorded. The trial was stopped arbitrarily at 30 seconds. This ability was measured at least twice per session, each measurement being preceded by a rest period of 30 seconds. All numbers represent the average of at least two trials. The difference between trials was always six seconds or less.

DRAWING The ability of each patient to draw Archimedes' screw was periodically observed.

\section{RESULTS}

EFFECT OF PHYSOSTIGMINE The length of time the patient could keep his tongue protruded was increased by physostigmine in 10 out of 12 patients. As shown in Table 2 the average duration of tongue extension was increased significantly by physostigmine, from a pretreatment average of 11.4 seconds (range 4-28 seconds) to 23.4 seconds (range $11-30$ seconds) after 30 minutes. The improvement began within five minutes. The maximum effect was seen between

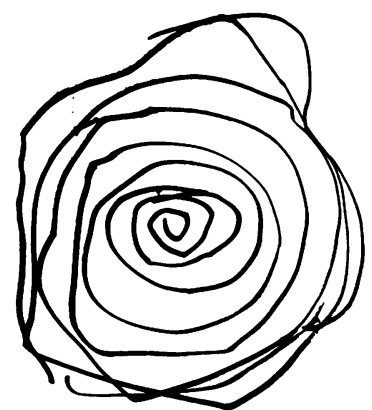

(a)

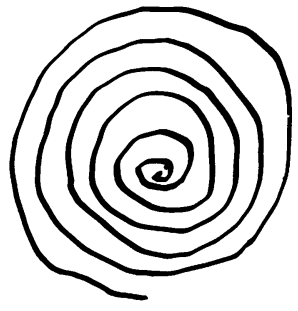

(b)
FIG. 1. The effect of physostigmine on drawing. (a) Before and (b) after the administration of $1 \mathrm{mg}$ physostigmine.

15 and 30 minutes, with a total duration of effect between 45 and 60 minutes. Edrophonium had no effect on tongue extension (Table 2). The ability to draw Archimedes' screw was tested in six patients (nos $3,4,7,8,11,12)$. This ability was improved by physostigmine in four patients nos $3,4,8,11)$. The effect of physostigmine on the ability of patient 3 to draw Archimedes' screw is shown in Fig. 1. The figure drawn before 


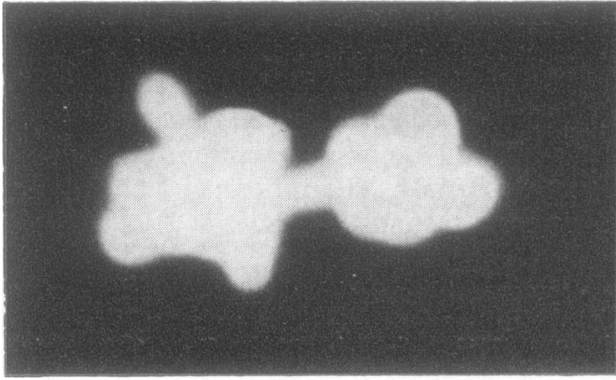

(a)

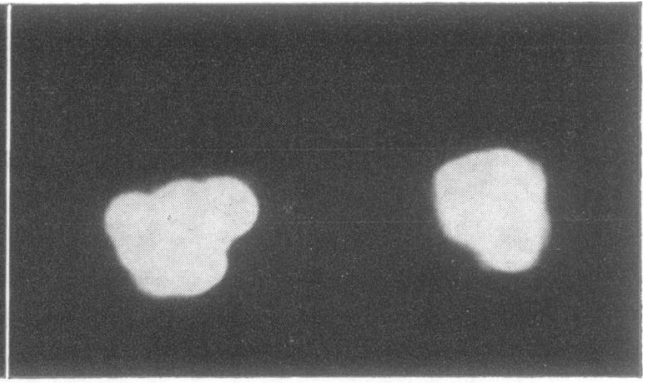

(b)

FIG. 2. The effect of physostigmine on limb chorea. (a) Before and (b) after the administration of $1 \mathrm{mg}$ physostigmine.

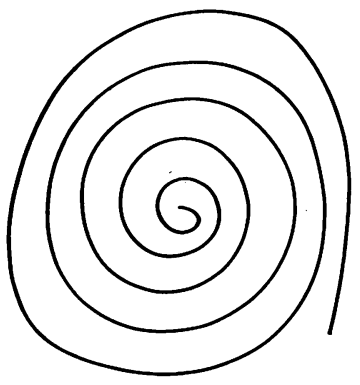

(a)

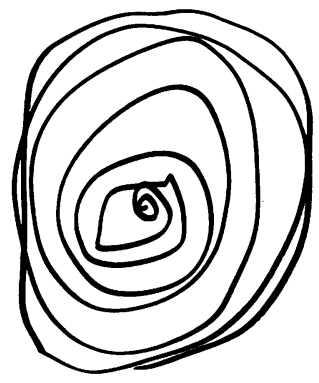

(b)
FIG. 3. The effect of scopolamine on drawing. (a) Before and (b) after the administration of $1 \mathrm{mg}$ scopolamine. the administration of physostigmine shows numerous interruptions of the smooth circular pattern not seen in those done after the admini- $\omega$ stration of physostigmine. Edrophonium given $\infty$ to four patients including patient 3 had no effeco $\bar{c}$ on this performance.

Physostigmine was observed to improve lim chorea in two out of six patients. The effect physostigmine on limb chorea in subject 3 is c shown in Fig. 2. Edrophonium had no observ क? able effect on limb chorea.

EFFECT OF SCOPOLAMINE The effect of scopolamine on tongue protrusion was tested only in the four patients who were able to maintain tongue protrusion relatively well. As shown in

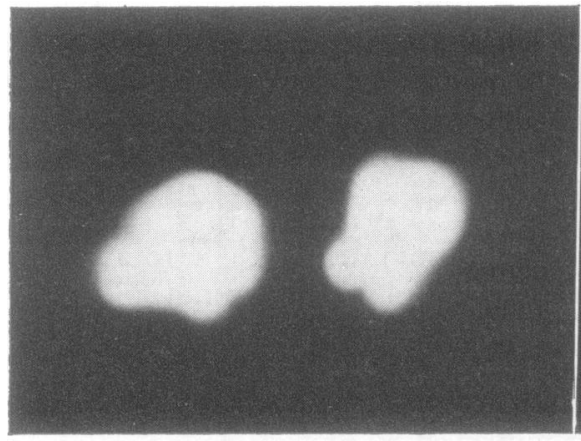

(a)

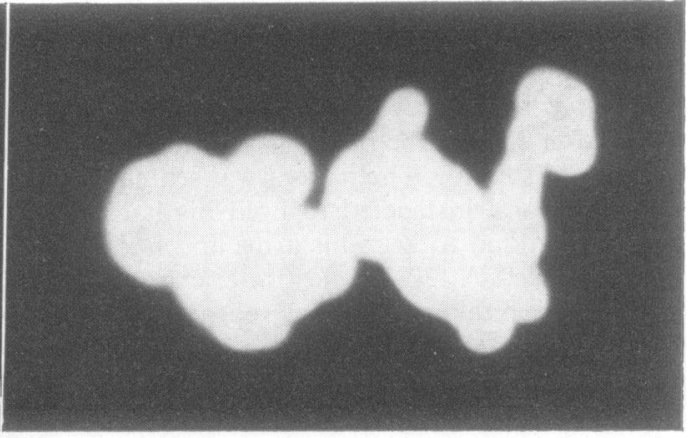

(b)

FIG. 4. The effect of scopolamine on limb chorea. (a) Before and (b) after the administration of $1 \mathrm{mg}$ scopolamine. 
Table 2, the average duration of tongue extension was 18.4 seconds before the administration of $1.0 \mathrm{mg}$ scopolamine. One hour after receiving scopolamine, tongue protrusion was decreased to an average of 6.3 seconds. The effect of scopolamine on the ability to draw the Archimedes' screw was observed in the six patients not tested for this ability with physostigmine. These six patients were free from upper extremity involvement in their disease and were able to perform the test well before the administration of the experimental medication. The patient's ability to draw the Archimedes' screw deteriorated in three of the six patients tested under the influence of $1.0 \mathrm{mg}$ scopolamine. This is illustrated in Fig. 3 which shows the effect of scopolamine on the ability of subject 2 to draw. The effect of scopolamine on limb chorea was also observed in the same six patients by the method described above. Scopolamine produced definite limb chorea in three out of six patients. An example of the effect of scopolamine on subject 2 is shown in Fig. 4 in which a definite increase in movement is demonstrated. Significantly, two of the three patients who developed limb chorea and were previously free from trunk movements also developed abnormal movements of the trunk during the period of peak efficacy of scopolamine. The abnormal movements elicited by scopolamine disappeared within three hours.

\section{DISCUSSION}

The results presented above suggest that alterations in the degree of central cholinergic activity may influence the severity of the abnormal movements in tardive dyskinesia. Physostigmine is a tertiary anticholinesterase that is able to cross the blood-brain barrier and increase the concentration of acetylcholine within the brain (Machne and Unna, 1963). Since edrophonium, a quarternary anticholinesterase that is unable to cross the blood-brain barrier, did not improve the dyskinetic movements, the physostigmineinduced alteration must reflect a central activity. Scopolamine, which worsened the dyskinesias, is a potent, centrally active acetylcholine antagonist (Ahmed and Marshall, 1962). These two agents have opposite effects on the cholinergic system, and opposite effects on the symptoms of tardive dyskinesia. The most likely site for these agents to act to influence these symptoms is the neostriatum. It is well accepted that choreiform movements are related to some sort of dysfunction of striatal neurones, specifically in the response of these neurones to dopamine (Bruyn, 1968; Birkmayer, 1969; Klawans, 1970, 1973a, b). The striatum contains the highest concentration of both dopamine (Bertler and Rosengren, 1959) and acetylcholine (Feldberg, 1945) found in the brain. Furthermore, dopamine and acetylcholine frequently have opposite effects on the neurones of the striatum (Klawans, 1973a). Neuroleptic agents, thought to be responsible for the production of tardive dyskinesia, act primarily on the striatum to block the dopaminergic receptors (Van Rossum, 1967). It is hypothesized that when the neuroleptic blockade is removed or diminished, the striatal neurones respond abnormally to the restored dopamine, resulting in the symptoms of tardive dyskinesia. The data presented here demonstrate that the acute administration of agents which alter the striatal influence of the cholinergic system can modify these dyskinesias.

Based upon studies of dopamine-related amphetamine-induced stereotyped behaviour in animals, it has been suggested that anticholinergics may both increase the severity of tardive dyskinesia in patients prone to this type of movement disorder and also increase the incidence of the disorder by altering the threshold for the appearance of these movements (Rubovits and Klawans, 1972). There is evidence that the incidence of tardive dyskinesia is greater in patients receiving both neuroleptics and anticholinergics than in patients receiving neuroleptics alone (Crane, 1971). This study provides some evidence to support the hypothesis that anticholinergic medication may be a predisposing factor in patients prone to the development of tardive dyskinesia. While all patients in this study had tardive dyskinesia and anticholinergic medication tended to worsen existing symptomatology, it was also found that in four patients with only lingual-facial-buccal dyskinesias anticholinergic medication brought out previously undetected trunkal and limb movements. There is at present no satisfactory explanation as to why lingualfacial-buccal movements are the most prominent manifestation of tardive dyskinesias. There is also no satisfactory explanation as to why anti- 
cholinergic agents elicit chorea in those individuals who manifest only lingual-facial-buccal dyskinesias. Anticholinergic medication does not cause these movements in normal volunteers (unpublished observations). Several factors may contribute to both of these phenomena. First, there may be regional variations in sensitivity within the caudate nucleus to the neurological alterations induced by neuroleptics, such that for a given level of physiological alteration, clinical manifestation of the derangement may vary from severe-for example, the face-to mild-for example, in the limbs-in the same patient. It is quite possible that those motor areas related to control of complex, fine movements of the face and hands may have more sensitive regulatory mechanisms than other areas, and that these areas may be more sensitive to any neurolepticinduced alteration in function. This may explain why lingual-facial-buccal movements are more frequently seen than trunkal movements. Alternatively, it is possible that the neuroleptics may alter the physiology of certain caudate areas more than that of other areas so that there may be a greater degree of neuroleptic-induced dysfunction in the facial area (manifested by lingualfacial-buccal movements) than in the trunk area. It is also possible that the face and hand areas are those most often clinically affected simply because their representations encompass a major proportion of the topography of the caudate, hence these caudatal areas would suffer the most damage.

In all of these proposed mechanisms there is a gradient of dysfunction between clinically manifest dysfunction and subclinical dysfunction. It is most probable that areas of the body which clinically appear to be symptom-free may be represented in the caudate nucleus by areas which may be significantly altered, needing only a small additional insult to cross the threshold between subclinical and clinically apparent dysfunction. In some cases, the addition of a small intravenous dose of an anticholinergic agent, which inhibited the cholinergic opposition to dopaminergic function, was sufficient 'additional insult' to allow previously undetected symptomatology to emerge. The acute administration of an anticholinergic agent to a patient with tardive dyskinesia may lower the threshold for the appearance of additional symptoms of the dis- order. This explains why anticholinergic medications may elicit trunkal or limb chorea in patients with lingual-facial-buccal movements and not in normal individuals. The concept that alterations related to limb chorea may be present in patients with tardive dyskinesia manifested only by lingual-facial-buccal movements is further supported by the fact that low dosage of levodopa has been shown to elicit severe limb and trunkal chorea in such patients (Klawans and McKendall, 1971; Klawans, 1973a). These same doses of levodopa do not elicit chorea in normal individuals. This apparent continuum of dysfunction from subclinical to clinical dyskinesias may also explain why the chronic administration of anticholinergic agents in conjunction with neuroleptic medications may create a greater risk for the development of tardive dyskinesia than neuroleptic medication alone. Amounts of dopamine which would be too small to produce clinical symptoms in patients who had not re

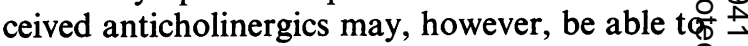
do so in those who had received anticholinergics $\frac{\text { 응 }}{3}$ It is suggested that the administration of antio cholinergics to patients undergoing long-ternt neuroleptic therapy be kept to a minimum.

\section{REFERENCES}

Ahmed, A., and Marshall, P. B. (1962). Relationship between anti-acetylcholine and anti-tremorine activity in antiParkinsonian and related drugs. British Journal of Pharmacology and Chemotherapy, 18, 247-254.

Bertler, A., and Rosengren, E. (1959). Occurrence and distribution of catechol amines in brain. Acta Physiologica Scandinavica, 47, 350-361.

Birkmayer, W. (1969). Der alpha-Methyl-P-Tyrosin-Effekt bei extrapyramidalen Erkrankungen. Wiener Klinische Wochenschrift, 81, 10-12.

Bruyn, G. W. (1968). Huntington's chorea. Historical, clinical and laboratory synopsis. In Handbook of Clinical Neurology, vol. 6, pp. 298-398. Edited by P. J. Vinken and G. W. Bruyn. North-Holland: Amsterdam.

Cotzias, G. C., Van Woert, M. H., and Schiffer, L. M. (1967). Aromatic amino acids and modification of Parkinsonism. New England Journal of Medicine, 276, 374-379.

Crane, G. E. (1968). Tardive dyskinesias in patients treated with major neuroleptics: a review of the literature. American Journal of Psychiatry, 124, no. 8, Suppl. 40-48.

Crane, G. (1971). Neuroleptics and proneness to motor disorders. Read before the American Psychiatric Association, $\mathbb{N}$ Columbus, Ohio.

Delay, J., and Deniker, P. (1969). Drug-induced extra- ? pyramidal syndromes. In Handbook of Clinical Neurology, $\mathrm{C}$ vol. 6, pp. 248-266. Edited by P. J. Vinken and G. W. Bruyn. North-Holland: Amsterdam. 
Duvoisin, R. C. (1967). Cholinergic-anticholinergic antagonism in parkinsonism. Archives of Neurology (Chic.), 17, 124-136.

Faurbye, A. (1970). The structural and biochemical basis of movement disorders in treatment with neuroleptic drugs and in extrapyramidal diseases. Comprehensive Psychiatry, 11, 205-225.

Feldberg, W. (1945). Present views on the mode of action of acetylcholine in the central nervous system. Physiological Reviews, 25, 596-642.

Huntington, G. (1872). On chorea. The Medical and Surgical Reporter, 26, 317-321.

Klawans, H. L. Jr (1968). The pharmacology of parkinsonism. Diseases of the Nervous System, 29, 805-816.

Klawans, H. L., Jr (1970). A pharmacologic analysis of Huntington's chorea. European Neurology, 4, 148-163.

Klawans, H. L., Jr (1973a). The Pharmacology of Extrapyramidal Movement Disorders. Karger: Basel.

Klawans, H. L., Jr (1973b). The pharmacology of tardive dyskinesias. American Journal of Psychiatry, 130, 82-86.

Klawans, H. L., Jr, Ilahi, M. M., and Shenker, D. (1970) Theoretical implications of the use of $\mathbf{L}$-dopa in parkinsonism. Acta Neurologica Scandinavica, 46, 409-441.
Klawans, H. L., Jr, and McKendall, R. R. (1971). Observations on the effect of levodopa on tardive lingual-facialbuccal dyskinesia. Journal of the Neurological Sciences, 14, 189-192.

Klawans, H. L., Jr, and Rubovits, R. (1972). Central cholinergic-anticholinergic antagonism in Huntington's chorea. Neurology (Minneap.), 22, 107-116.

Machne, X., and Unna, K. R. W. (1963). Actions at the central nervous system. In Heffter's Handbuch der experimentellen Pharmakologie. Suppl. 15. Cholinesterases and anticholinesterase agents. pp. 679-700. Springer: Berlin.

Paulson, G. W. (1968). 'Permanent' or complex dyskinesias in the aged. Geriatrics, 23, no. 9, 105-110.

Rubovits, R., and Klawans, H. L., Jr (1972). Implications of amphetamine-induced stereotyped behavior as a model for tardive dyskinesias. Archives of General Psychiatry, 27, 502-507.

Van Rossum, J. M. (1967). The significance of dopaminereceptor blockade for the action of neuroleptic drugs. In Neuro-Psycho-Pharmacology. Proceedings of the Fifth International Congress of the Collegium Internationale Neuro-Psychopharmacologicum, Washington, 1966, pp. 321-329. International Congress Series No. 129. Excerpta Medica: Amsterdam. 